\title{
Instantaneous identification of localized non-linearities in steel framed structures
}

\author{
Pierre Argoul ${ }^{2}$, Rosario Ceravolo $^{1, a}$, G.V. Demarie ${ }^{1}$ and D. Sabia ${ }^{1}$ \\ 1 Dip. Ingegneria Strutturale e Geotecnica, Politecnico di Torino, c. Duca degli Abruzzi 24, 10129 Torino, Italy \\ 2 Université Paris-Est, UR Navier, École des Ponts ParisTech, 6 \& 8 avenue Blaise Pascal, Champs-sur-Marne, \\ 77455 Marne-la-Vallée Cedex 2, France
}

Received 2 October 2008, Accepted 1 December 2009

\begin{abstract}
This paper discusses the characteristics of time-frequency estimators to be used in the identification of systems with localized non-linearities. The common idea underlying this research is that, for certain classes of structural response signals, the availability of a limited number of experimental data can be partially obviated by taking into account the "localisation" in time of the frequency components of the signals. Time-frequency techniques for structural identification are reported that extend the definition of instantaneous time-frequency estimators and Gabor instantaneous estimators were extracted from nonstationary vibration signals. In order to foresee their validity on the basis of measured data, methods were applied to seismic responses obtained from numerical tests conducted on steel frames. The results obtained made it possible to evaluate the characteristics of time-frequency identification techniques as well as their efficiency when applied to non-linear structures.
\end{abstract}

Key words: Non-linear identification / Volterra series / framed structures / steel structures / instantaneous frequency / time-frequency analysis

Résumé - Identification instantanée de non-linéarités locales dans les portiques métalliques. Cet article s'intéresse aux propriétés des estimateurs temps-fréquence utilisés dans l'identification de systèmes mécaniques avec des non-linéarités localisées. L'idée de base est que pour certaines classes de réponses des structures, on peut suppléer au nombre limité de données expérimentales en prenant en compte la localisation en temps et en fréquence du signal analysé. Des techniques tempsfréquence qui étendent la notion d'estimateurs instantanés temps-fréquence au cas de comportement non linéaire des structures ont été développées à des fins d'identification de paramètres. L'analyse en gaborettes est appliquée dans le cas de réponses sous excitation non stationnaire. Afin d'étudier leur performance et de définir leur domaine de validité, les méthodes d'identification proposées ont été appliquées aux cas de réponses de portiques métalliques sous excitations sismiques, calculées par des modèles numériques. Les résultats obtenus permettent de préciser les caractéristiques intrinsèques des méthodes d'identification en temps-fréquence et d'évaluer leur efficacité dans l'identification non-linéaire des structures.

Mots clés : Identification non-linéaire / série de Volterra / structures portiques / structures métalliques / fréquence instantanée / analyse temps-fréquence

\section{Introduction}

In applications requiring the identification of special features of the dynamic response or a reliable evaluation of safety conditions, the assumption of linear behaviour might be insufficient. In structural steel frameworks, for instance, a potential source of non-linearity is located in beam-column connections and in basic elements forming integrated parts of the steel frame $[1,2]$. In multi-story buildings, a non-linear behaviour may also be induced by the interaction with non-structural elements, such as infill

\footnotetext{
${ }^{a}$ Corresponding author: rosario.ceravolo@polito.it
}

panels that participate in resisting dynamically induced loads [3].

All the mentioned phenomena call for the use of specific signal processing tools to extract reliable information about non-linear effects. This is particularly true in some specific structural engineering applications, such as passive and active control or diagnostics, where models are required to capture and reproduce even weak non-linear features in the dynamic response.

Several identification techniques for linear systems require the input to be known or stationary and are based on fitting a frequency response function $H_{1}$ [4]. When 


\section{Nomenclature}

\begin{tabular}{|c|c|}
\hline$[C]$ & damping matrix \\
\hline$D_{j}\left(n^{*}, m\right)$ & $\begin{array}{l}\text { value of the STFT at discrete time instant } n^{*} \Delta t \text { and at frequency } \\
m \Delta f \text { of the measured system response at the } j \text { th d.o.f. }\end{array}$ \\
\hline$D_{V, j}\left(n^{*}, m\right)$ & $\begin{array}{l}\text { value of the STFT at discrete time instant } n^{*} \Delta t \text { and at frequency } \\
m \Delta f \text { of the Volterra series expansion at the } j \text { th d.o.f. }\end{array}$ \\
\hline$D^{(j)}(t, f)$ & Short-Time Fourier Transform of system output at location $j$ \\
\hline$D_{n}^{(j)}(t, f)$ & Short-Time Fourier Transform of the $n$th Volterra term \\
\hline$\{F\}_{\mathrm{nl}}$ & vector of internal non-linear forces \\
\hline$\{F\}_{\mathrm{nl}, x}$ & vector of the $x$-components of the non-linear forces \\
\hline$\{F\}_{\mathrm{nl}, y}$ & vector of the $y$-components of the non-linear forces \\
\hline$F_{\mathrm{ob}}\left(n^{*}, \boldsymbol{p}\right)$ & objective function \\
\hline$g_{n}\left[\{x\}_{1},\{x\}_{2}, \ldots,\{x\}_{n-1}\right]$ & functional depending on the form of non-linearity \\
\hline$h_{n}^{(j: \text { aаa...aa })}\left(t_{1}, \ldots, t_{n}\right)$ & direct higher-order impulse response function \\
\hline$h_{n}^{(j: \mathrm{aa} \ldots \mathrm{ab} \ldots \mathrm{bb})}\left(\tau_{1}, \ldots, \tau_{n}\right)$ & cross higher-order impulse response function \\
\hline$[K]$ & stiffness matrix \\
\hline$[M]$ & mass matrix \\
\hline$k_{3 x}, k_{3 y}$ & $\begin{array}{l}\text { cubic stiffness coefficients corresponding to the first interstory drift } \\
\text { respectively for } x \text { and } y \text { directions }\end{array}$ \\
\hline$\{M\}_{\mathrm{nl}, \mathrm{O}}$ & $\begin{array}{l}\text { vector collecting the moments of the internal non-linear forces } \\
\text { with respect the origin of the axes }\end{array}$ \\
\hline$N$ & number of storys of the building \\
\hline$n_{m}$ & number of points where the system vibration has been measured \\
\hline$\{p\}$ & vector of modal coordinates \\
\hline$\{p\}_{\text {red }}$ & response vector of the reduced modal model \\
\hline$p$ & vector of the unknown dynamic properties of the system \\
\hline$p_{\mathrm{nl}}$ & vector containing the dynamic non-linear parameters of the system \\
\hline$\{q\}$ & vector of displacements of the multi-story building \\
\hline$\left\{\ddot{q}_{s}\right\}$ & vector of seismic accelerations (components parallel to $x$ and $y$ axes) \\
\hline$[R]$ & matrix of non-linearity (non-diagonal) \\
\hline$[R]_{\mathrm{red}}$ & reduced matrix of non-linear coefficients \\
\hline$[t]$ & matrix which applies the seismic input to the degrees of freedom \\
\hline$\{u\},\{v\}$ and $\{\gamma\}$ & $\begin{array}{l}N \times 1 \text { vectors collecting the translations parallel to } \\
x \text { and } y \text { axis and the floor rotations }\end{array}$ \\
\hline$\{u\}$ & vector of the external inputs acting on the system \\
\hline$u^{(\mathrm{a})}(t), u^{(\mathrm{b})}(t)$ & inputs applied to points $\mathrm{a}$ and $\mathrm{b}$ \\
\hline$w(t)$ & running window function \\
\hline$\{x(t)\}$ & vector of displacements \\
\hline$x_{n}^{(j)}(t)$ & $n$th order contribution to the structural response at the $j$ th d.o.f. \\
\hline$\{x(t)\}_{n}$ & $\begin{array}{l}n \text {th order contribution of the Volterra series expansion } \\
\text { to the system's response }\end{array}$ \\
\hline$[\alpha],[\beta]$ & matrices of the non-linear stiffness coefficients \\
\hline$[\Gamma]$ & matrix of modal participation factors \\
\hline$[\Phi]$ & normalized modal matrix \\
\hline$\left\{\Phi_{i}\right\}$ & $i$ th row of the normalized modal matrix $[\Phi]$ \\
\hline$\left\{\Phi_{i}\right\}_{\text {red }}$ & $i$ th row of the reduced modal matrix \\
\hline$[2 \zeta \omega]$ & modal damping matrix (diagonal) \\
\hline$\left[\omega^{2}\right]$ & modal frequencies matrix \\
\hline$\left[\omega^{2}\right],[2 \zeta \omega]$ & matrices of modal frequencies and damping \\
\hline$\left[\omega^{2}\right]_{\mathrm{red}},[2 \zeta \omega]_{\mathrm{red}}$ & reduced matrices of modal frequencies and damping \\
\hline
\end{tabular}


excitation is unknown and non-stationary, in mechanical systems we can still rely on local stationarity [5-7] and may appeal to instantaneous curve-fitting procedures in order to define punctual estimators of the local dynamic features [8-10]. In this connection, time-frequency analysis has recently opened new prospects, and its efficacy in non-linear identification may be enhanced by assigning it a role within the framework of the Volterra series representation. Important advantages in using the Volterra series are the graduation of non-linear effects and stability with respect to added noise.

This paper applies a technique for structural identification that extends the definition of instantaneous time-frequency estimators to systems whose input/output relationship is depicted as a Volterra series [11, 12]. The theoretical background outlined for elementary systems [13], here was applied to more complex structures, also in order to support applications and to be potentially applied to the identification of real framed structures, including infilled frames with non-linearities in the interstory stiffness.

A numerical application to the seismic response of a three dimensional 3D steel frame with localized nonlinearity is finally described. Non-linearity is characterised by polynomial stiffness and is supposed to be concentrated in the joints between steel members, in order to simulate their flexibility and possibly an incipient damage. To this end, it is assumed that the input/output relationship to be identified is approximated by a certain number of terms of the Volterra polynomial representation. The structure was ideally subjected to an earthquake loading.

\section{The Volterra series representation for MDOF systems}

Let us consider a Multiple Degree of Freedom (MDOF) non-linear system having $N$ degrees of freedom whose motion can be described by the following equation:

$$
[M]\{\ddot{x}\}+[C]\{\dot{x}\}+[K]\{x\}+\{F\}_{n l}=\{u\}
$$

where $[M],[C]$ and $[K]$ are respectively the mass, damping and stiffness matrices of the underlying linear system; $\{x(t)\}$ is the system response; $\{u\}$ is the input; $\{F\}_{\mathrm{nl}}$ is the vector of non-linear forces, which, in general, takes into account non-linearity both in the damping mechanism and in the restoring forces.

If the system allows a Volterra series representation, then the output at the $j$ th degree of freedom can be expressed as an infinite summation of terms [14-18]:

$$
x^{(j)}(t)=x_{1}^{(j)}(t)+x_{2}^{(j)}(t)+x_{3}^{(j)}(t)+\ldots=\sum_{n=1}^{+\infty} x_{n}^{(j)}(t)
$$

The term $x_{n}^{(j)}(t)$ represents the $n$th order contribution to the global response of the system at the $j$ th d.o.f. and can be calculated via a $n$-fold convolution of the inputs acting on the system and the higher-order impulse response functions (HIRFs). For example, if a system is excited in two location points, a and b, then the following expression holds:

$$
\begin{aligned}
& x_{n}^{(j)}(t)=\int_{-\infty}^{+\infty} \int_{-\infty}^{+\infty} \ldots \int_{-\infty}^{+\infty} h_{n}^{(j: \mathrm{aa} \ldots \mathrm{aa})}\left(\tau_{1}, \ldots, \tau_{n}\right) \\
& \times u^{(\mathrm{a})}\left(t-\tau_{1}\right) \ldots u^{(\mathrm{a})}\left(t-\tau_{n}\right) \mathrm{d} \tau_{1} \ldots \mathrm{d} \tau_{n}+ \\
& +\int_{-\infty}^{+\infty} \int_{-\infty}^{+\infty} \ldots \int_{-\infty}^{+\infty} h_{n}^{(j: \mathrm{aa} \ldots \mathrm{ab})}\left(\tau_{1}, \ldots, \tau_{n}\right) u^{(\mathrm{a})}\left(t-\tau_{1}\right) \ldots \\
& \quad \times u^{(\mathrm{b})}\left(t-\tau_{n}\right) \mathrm{d} \tau_{1} \ldots \mathrm{d} \tau_{n}+\ldots \\
& \ldots+\int_{-\infty}^{+\infty} \int_{-\infty}^{+\infty} \ldots \int_{-\infty}^{+\infty} h_{n}^{(j: \mathrm{aa} \ldots \mathrm{bb})}\left(\tau_{1}, \ldots, \tau_{n}\right) u^{(\mathrm{a})}\left(t-\tau_{1}\right) \ldots \\
& \quad \times u^{(\mathrm{b})}\left(t-\tau_{n}\right) \mathrm{d} \tau_{1} \ldots \mathrm{d} \tau_{n}+\ldots \\
& \ldots+\int_{-\infty}^{+\infty} \ldots \int_{-\infty}^{+\infty} h_{n}^{(j: \mathrm{bb} \ldots \mathrm{bb})}\left(\tau_{1}, \ldots, \tau_{n}\right) u^{(\mathrm{b})}\left(t-\tau_{1}\right) \ldots \\
& \quad \times u^{(\mathrm{b})}\left(t-\tau_{n}\right) \mathrm{d} \tau_{1} \ldots \mathrm{d} \tau
\end{aligned}
$$

where $u^{(\mathrm{a})}(t)$ and $u^{(\mathrm{b})}(t)$ are the inputs applied to points $\mathrm{a}$ and $\mathrm{b}, h_{n}^{(j \text { :aa...aa) }}\left(t_{1}, \ldots, t_{n}\right)$ is a direct HIRF (it gives the contribution of the input $u^{(\mathrm{a})}(t)$, considered $n$ times), $h_{n}^{(j \text { :aa...ab...bb) }}\left(\tau_{1}, \ldots, \tau_{n}\right)$ is a cross-HIRF, which gives the contribution of the inputs $u^{(\mathrm{a})}(t)$, considered $n_{\mathrm{a}}$ times, and $u^{(\mathrm{b})}(t)$, considered $n_{\mathrm{b}}$ times $\left(n_{\mathrm{a}}+n_{\mathrm{b}}=n\right)$.

A dual representation of Equation (1) exists in the frequency domain, where system dynamics can be described by direct and cross higher-order frequency response functions (HFRFs). Furthermore, a convergence condition must be associated for the Volterra series representation to be meaningful, generally involving some limitations on the amplitude of the input and the time interval on which the functional expansion is calculated $[19,20]$. The analytical expressions of the direct and cross HFRFs can be calculated via the harmonic probing algorithm [14].

Alternatively, it has been shown [21] that the output of a Volterra system can be calculated by solving an infinite series of associated linear equations (ALEs), the first of which represents the equation of motion of the underlying linear system, for a MDOF system, one obtains the following set of associated linear systems (ALSs):

$$
\begin{aligned}
& \left\{\begin{array}{l}
{[M]\{\ddot{x}\}_{1}+[C]\{\dot{x}\}_{1}+[K]\{x\}_{1}=\{u\}} \\
{[M]\{\ddot{x}\}_{2}+[C]\{\dot{x}\}_{2}+[K]\{x\}_{2}=g_{2}\left[\{x\}_{1}\right]} \\
{[M]\{\ddot{x}\}_{3}+[C]\{\dot{x}\}_{3}+[K]\{x\}_{3}=g_{3}\left[\{x\}_{1},\{x\}_{2}\right]} \\
\ldots \\
{[M]\{\ddot{x}\}_{n}+[C]\{\dot{x}\}_{n}+[K]\{x\}_{n}=g_{n}\left[\{x\}_{1}\right.} \\
\left.\{x\}_{2}, \ldots,\{x\}_{n-1}\right] \\
\cdots \\
\{x\}=\{x\}_{1}+\{x\}_{2}+\{x\}_{3}+\ldots+\{x\}_{n}+\ldots
\end{array}\right.
\end{aligned}
$$


where $g_{n}\left[\{x\}_{1},\{x\}_{2}, \ldots,\{x\}_{n-1}\right]$ is a functional depending on the form of non-linearity. From Equation (4), it is possible to determine the $n$th order contribution of the Volterra series expansion, $\{x(t)\}_{n}$, as the response of the underlying linear system to an input which is a non-linear function of the first $n$ - 1 terms.

\section{Instantaneous identification of a MDOF system with Volterra non-linearity}

If the output of a system is nonstationary, the frequency content of the vibration changes over time and, consequently, its time-frequency representation leads to the definition of instantaneous characteristics. A classical example is instantaneous frequency, whose meaning inspired far-reaching discussions in the field of signal analysis (see the extensive literature on this theme, from Carson [22] to Cohen [6]).

Instantaneous estimation will depend on a choice of time-varying spectrum. For instance, by applying the definition of the short-time Fourier transform (STFT) [6] to the Volterra series expansion of the input/output relationship, as expressed by Equation (1), the following relationship is obtained:

$$
\begin{aligned}
D^{(j)}(t, f) & =\int_{-\infty}^{+\infty} x^{(j)}(\tau) w(\tau-t) \mathrm{e}^{i 2 \pi f \tau} \mathrm{d} \tau \\
& =\int_{-\infty}^{+\infty}\left(x_{1}^{(j)}(\tau)+x_{2}^{(j)}(\tau)+\ldots\right) w(\tau-t) \mathrm{e}^{i 2 \pi f \tau} \mathrm{d} \tau \\
& =D_{1}^{(j)}(t, f)+D_{2}^{(j)}(t, f)+\ldots
\end{aligned}
$$

where $D^{(j)}(t, f)$ is the STFT of system output at location $j, D_{n}^{(j)}(t, f)$ is the STFT of the $n$th Volterra term, and $w(t)$ is a running window function, defined in the time domain and centred around time $t$. If the analytical forms of the direct and cross-HIRFs (or, in the frequency domain, of the HFRFs) are available, then $D^{(j)}(t, f)$ can be calculated directly by introducing Equation (3) into Equation (5).

Equations (4) and (5) constitute the basis for a parametric identification algorithm: let $\boldsymbol{p}$ be the vector of parameters that describe the dynamic properties of the system, including linear and non-linear parameters; let us suppose that system vibration has been measured in $n_{m}$ points and let us introduce the following instantaneous objective function:

$$
F_{\mathrm{ob}}\left(n^{*}, \boldsymbol{p}\right)=\left.\sum_{j=1}^{n_{m}} \sum_{m=0}^{M-1}|| D_{j}\left(n^{*}, m\right)\right|^{2}-\left|D_{V, j}\left(n^{*}, m, \boldsymbol{p}\right)\right|^{2} \mid
$$

where $D_{j}\left(n^{*}, m\right)$ and $D_{V, j}\left(n^{*}, m, \boldsymbol{p}\right)$ are the values of the STFTs at discrete time instant $n^{*} \Delta t$ and at frequency $m \Delta f$ of the system response at the $j$ th d.o.f., as measured and calculated through the Volterra series expansion (Eq. (4)) for a given configuration of parameters $\boldsymbol{p}$.
The number of frequency samples, $M$, to be considered in the objective function in Equation (6) will depend on the frequency range under analysis.

When working with specific representations that satisfy the time marginal property [6], $F_{\text {ob }}$ gives the difference between the instantaneous energies of the experimental signals (measured at locations $1,2, \ldots n_{m}$ ) and of the system output corresponding to a given configuration of the unknown parameters $\boldsymbol{p}$ : by resorting to classical optimisation procedures, it proves possible to determine instant by instant the minimum of $F_{\mathrm{ob}}\left(n^{*}, \boldsymbol{p}\right)$, which defines the vector of instantaneous estimates, $\boldsymbol{p}(t)$. A final estimate of the system's parameters is then possible on the basis of $\boldsymbol{p}(t)$. Notice that, the STFT being a linear transform, the optimisation expressed by Equation (6) can also be conducted separately for each component $D_{n}^{(j)}(t, f)$ associated with the $n$th Volterra term.

If the vibration of the system is determined by a limited number of modes $n_{\text {red }}<N$, and, without losing generality, the linear part of the damping mechanism is written in the classical form, the ALEs assume the following expression in the modal space:

$$
\begin{aligned}
& \left\{\begin{array}{l}
\{\ddot{p}\}_{1, \text { red }}+[2 \zeta \omega]_{\mathrm{red}}\{\dot{p}\}_{1, \text { red }}+\left[\omega^{2}\right]_{\text {red }}\{p\}_{1, \text { red }} \\
=[\Phi]_{\text {red }}^{T}\{u\} \\
\{\ddot{p}\}_{2, \text { red }}+[2 \zeta \omega]_{\text {red }}\{\dot{p}\}_{2, \text { red }}+\left[\omega^{2}\right]_{\text {red }}\{p\}_{2, \text { red }} \\
\quad=g_{2}\left[\{p\}_{1, \text { red }}\right] \\
\{\ddot{p}\}_{3, \text { red }}+[2 \zeta \omega]_{\text {red }}\{\dot{p}\}_{3, \text { red }}+\left[\omega^{2}\right]_{\text {red }}\{p\}_{3, \text { red }} \\
\quad=g_{3}\left[\{p\}_{1, \text { red }},\{p\}_{2, \text { red }}\right] \\
\ldots \\
\{\ddot{p}\}_{n, \text { red }}+[2 \zeta \omega]_{\text {red }}\{\dot{p}\}_{n, \text { red }}+\left[\omega^{2}\right]_{\text {red }}\{p\}_{n, \text { red }} \\
\quad=g_{n}\left[\{p\}_{1, \text { red }},\{p\}_{2, \text { red }}, \ldots\{p\}_{n-1, \text { red }}\right] \\
\ldots \\
\{x\} \cong[\Phi]_{\text {red }}\left(\{p\}_{1, \text { red }}+\{p\}_{2, \text { red }}+\{p\}_{3, \text { red }}+\ldots\right)
\end{array}\right.
\end{aligned}
$$

where $\{p\}_{\text {red }}$ and $[\Phi]_{\text {red }}$ are the response vector and the modal matrix associated with the reduced modal model, respectively. In this case, Equations (5) and (7) will constitute the basis for the parametric identification algorithm. The vector of the dynamic parameters to be identified is now: $\boldsymbol{p}=\left\{\zeta_{1} \ldots \zeta_{n} \omega_{1} \ldots \omega_{n} \boldsymbol{p}_{\mathrm{nl}}\right\}^{\mathrm{T}}$, where $\boldsymbol{p}_{\mathrm{nl}}$ contains all the non-linear parameters; if the vibration has been measured at $n_{m}$ points, the instantaneous estimators are still obtained from the minimization of the objective function (Eq. (6)).

\section{Instantaneous identification of buildings with weak non-linearity in the interstory-drift}

A typical multi-story building is characterised by a system of orthogonal frames, as depicted in Figure 1. The 

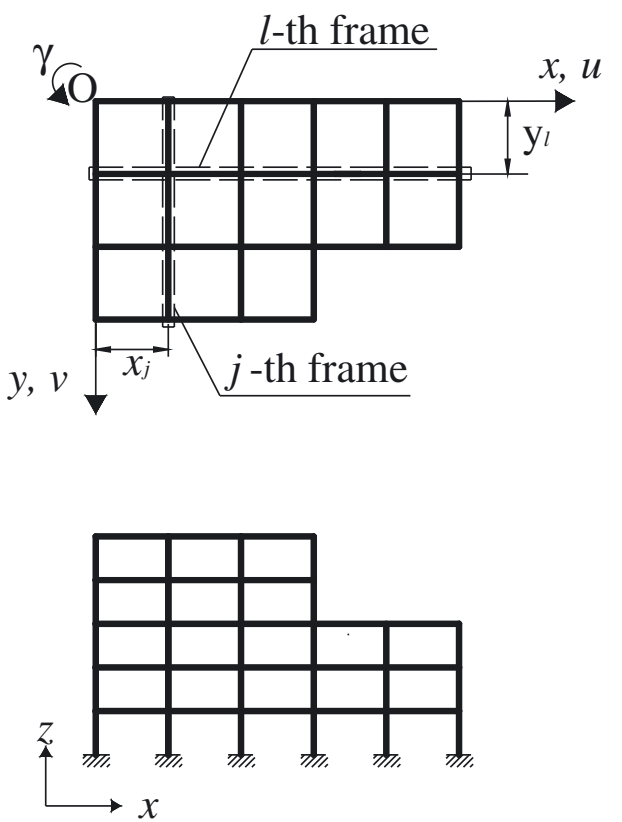

Fig. 1. Three-dimensional frame (conventions on geometry and displacements).

equation of motion of the structure subjected to a seismic excitation along the two horizontal axes can be written under the following assumptions:

- the masses are lumped at each floor;

- diaphragmatic behaviour at the story level;

- all the members are axially rigid;

- the torsional stiffness of the members is neglected;

- all the beams retain a linear behaviour;

- in order to simulate a mild damage, springs with polynomial non-linearity are introduced between adjacent stories (one for each frame), simulating a non-linear law depending on the floor drift.

In the identification of real buildings, the validity of the above assumptions should be checked and the contribution of non-structural elements (e.g. infilled walls) must be incorporated into the model.

By considering only three degrees of freedom per story, namely the two translations and a floor rotation, the dynamic equilibrium is assumed to be of the form:

$$
[M]\{\ddot{q}\}+[C]\{\dot{q}\}+[K]\{q\}+\{F\}_{\mathrm{nl}}=-[M][t]\left\{\ddot{q}_{\mathrm{s}}\right\}
$$

where $\{q\}$ is the global vector of displacements, $\left\{\ddot{q}_{\mathrm{s}}\right\}$ is the vector of seismic accelerations (components parallel to $x$ and $y$ axes) and $[t]$ is a matrix which applies the seismic input to the degrees of freedom:

$$
\begin{aligned}
& \{q\}=\left\{\begin{array}{l}
\{u\} \\
\{v\} \\
\{\gamma\}
\end{array}\right\}, \quad\left\{\ddot{q}_{\mathrm{s}}\right\}=\left\{\begin{array}{l}
\ddot{x}_{\mathrm{s}} \\
\ddot{y}_{\mathrm{s}}
\end{array}\right\}, \\
& {[t]^{\mathrm{T}}=\left[\begin{array}{llllllllllll}
1 & 1 & \ldots & 1 & 0 & 0 & \ldots & 0 & 0 & \ldots & 0 \\
0 & 0 & \ldots & 0 & 1 & 1 & \ldots & 1 & 0 & 0 & \ldots & 0
\end{array}\right]}
\end{aligned}
$$

$\{u\},\{v\}$ and $\{\gamma\}$ being $N \times 1$ vectors collecting the translations parallel to $x$ and $y$ axis and the floor rotations respectively of the $N$ stories.

It is assumed that, for each frame, non-linear internal forces can be expressed as a polynomial function of the inter-story displacement, which can generally be parametrically related to structural damage; for example, if nonlinearity is represented by $\alpha_{i, l}$ and $\beta_{i, j}$ parameters, which are the cubic stiffness coefficients associated with the $l$-th frame parallel to $x$-axis and with the $j$ th frame parallel to $y$-axis, below the $i$ th floor, then $\{F\}_{\mathrm{nl}}$ will assume the following form:

$$
\begin{aligned}
& \{F\}_{\mathrm{nl}}=\left\{\begin{array}{c}
\{F\}_{\mathrm{nl}, x} \\
\{F\}_{\mathrm{nl}, y} \\
\{M\}_{\mathrm{nl}, \mathrm{O}}
\end{array}\right\} \\
& \{F\}_{\mathrm{nl}, x}= \\
& \sum_{l=1}^{N_{x}}\left\{\begin{array}{c}
\alpha_{1, l}\left(u_{1}-\gamma_{1} y_{l}\right)^{3}-\alpha_{2, l}\left[\left(u_{2}-u_{1}\right)\right. \\
\left.-\left(\gamma_{2}-\gamma_{1}\right) y_{l}\right]^{3} \\
\alpha_{2, l}\left[\left(u_{2}-u_{1}\right)-\left(\gamma_{2}-\gamma_{1}\right) y_{l}\right]^{3} \\
-\alpha_{3, l}\left[\left(u_{3}-u_{2}\right)-\left(\gamma_{3}-\gamma_{2}\right) y_{l}\right]^{3} \\
\ldots \\
\alpha_{N, l}\left[\left(u_{N}-u_{N-1}\right)-\left(\gamma_{N}-\gamma_{N-1}\right) y_{l}\right]^{3}
\end{array}\right\} \\
& =\sum_{l=1}^{N_{x}}\{F\}_{\mathrm{nl}, x}^{l}
\end{aligned}
$$$$
\{F\}_{\mathrm{nl}, y}=
$$

$$
\sum_{j=1}^{N_{y}}\left\{\begin{array}{c}
\beta_{1, j}\left(v_{1}+\gamma_{1} x_{j}\right)^{3}-\beta_{2, j}\left[\left(v_{2}-v_{1}\right)\right. \\
\left.+\left(\gamma_{2}-\gamma_{1}\right) x_{j}\right]^{3} \\
\beta_{2, j}\left[\left(v_{2}-v_{1}\right)+\left(\gamma_{2}-\gamma_{1}\right) x_{j}\right]^{3} \\
-\beta_{3, j}\left[\left(v_{3}-v_{2}\right)+\left(\gamma_{3}-\gamma_{2}\right) x_{j}\right]^{3} \\
\ldots \\
\beta_{N, j}\left[\left(v_{N}-v_{N-1}\right)+\left(\gamma_{N}-\gamma_{N-1}\right) x_{j}\right]^{3}
\end{array}\right\}
$$

$$
=\sum_{j=1}^{N_{y}}\{F\}_{\mathrm{nl}, y}^{j}
$$

$$
\{M\}_{\mathrm{nl}, \mathrm{O}}=-\sum_{l=1}^{N_{x}}\{F\}_{\mathrm{nl}, x}^{l} y_{l}+\sum_{j=1}^{N_{y}}\{F\}_{\mathrm{nl}, y}^{j} x_{j}
$$

where $N_{x}, N_{y}$ stand for the number of frames parallel to the $x$ and $y$ axes, respectively; $\{F\}_{\mathrm{nl}, x},\{F\}_{\mathrm{nl}, y}$ and $\{M\}_{\mathrm{nl}, \mathrm{O}}$ are the vectors collecting the $x$ - and $y$ components of the internal non-linear forces and their moments with respect to the origin of the axes. 


$$
\begin{aligned}
& {[R]=[\Phi] \cdot\left[\begin{array}{c}
{[\alpha][A]} \\
{[\beta][B]} \\
-[\alpha][D]+[\beta][E]
\end{array}\right]} \\
& {[A]=\left[\begin{array}{c}
\left\{A_{1}\right\} \\
\left\{A_{2}\right\} \\
\ldots \\
\left\{A_{N}\right\}
\end{array}\right],[B]=\left[\begin{array}{c}
\left\{B_{1}\right\} \\
\left\{B_{2}\right\} \\
\ldots \\
\left\{B_{N}\right\}
\end{array}\right],[D]=\left[\begin{array}{c}
\left\{D_{1}\right\} \\
\left\{D_{2}\right\} \\
\ldots \\
\left\{D_{N}\right\}
\end{array}\right],[E]=\left[\begin{array}{c}
\left\{E_{1}\right\} \\
\left\{E_{2}\right\} \\
\ldots \\
\left\{E_{N}\right\}
\end{array}\right]} \\
& \left\{A_{i}\right\}=\sum_{l=1}^{N_{x}}\left[\left(\left\{\Phi_{i}\right\}-\left\{\Phi_{i-1}\right\}\right)-\left(\left\{\Phi_{2 N+i}\right\}-\left\{\Phi_{2 N+i-1}\right\}\right) y_{l}\right] \otimes\left[\left(\left\{\Phi_{i}\right\}-\left\{\Phi_{i-1}\right\}\right)-\left(\left\{\Phi_{2 N+i}\right\}-\left\{\Phi_{2 N+i-1}\right\}\right) y_{l}\right] \\
& \otimes\left[\left(\left\{\Phi_{i}\right\}-\left\{\Phi_{i-1}\right\}\right)-\left(\left\{\Phi_{2 N+i}\right\}-\left\{\Phi_{2 N+i-1}\right\}\right) y_{l}\right] \\
& \left\{B_{i}\right\}=\sum_{j=1}^{N_{y}}\left[\left(\left\{\Phi_{N+i}\right\}-\left\{\Phi_{N+i-1}\right\}\right)+\left(\left\{\Phi_{2 N+i}\right\}-\left\{\Phi_{2 N+i-1}\right\}\right) x_{j}\right] \otimes\left[\left(\left\{\Phi_{N+i}\right\}-\left\{\Phi_{N+i-1}\right\}\right)+\left(\left\{\Phi_{2 N+i}\right\}-\left\{\Phi_{2 N+i-1}\right\}\right) x_{j}\right] \\
& \otimes\left[\left(\left\{\Phi_{N+i}\right\}-\left\{\Phi_{N+i-1}\right\}\right)+\left(\left\{\Phi_{2 N+i}\right\}-\left\{\Phi_{2 N+i-1}\right\}\right) x_{j}\right] \\
& \left\{D_{i}\right\}=\sum_{l=1}^{N_{x}}\left[\left(\left\{\Phi_{i}\right\}-\left\{\Phi_{i-1}\right\}\right)-\left(\left\{\Phi_{2 N+i}\right\}-\left\{\Phi_{2 N+i-1}\right\}\right) y_{l}\right] \otimes\left[\left(\left\{\Phi_{i}\right\}-\left\{\Phi_{i-1}\right\}\right)-\left(\left\{\Phi_{2 N+i}\right\}-\left\{\Phi_{2 N+i-1}\right\}\right) y_{l}\right] \\
& \otimes\left[\left(\left\{\Phi_{i}\right\}-\left\{\Phi_{i-1}\right\}\right)-\left(\left\{\Phi_{2 N+i}\right\}-\left\{\Phi_{2 N+i-1}\right\}\right) y_{l}\right] y_{l} \\
& \left\{E_{i}\right\}=\sum_{j=1}^{N_{y}}\left[\left(\left\{\Phi_{N+i}\right\}-\left\{\Phi_{N+i-1}\right\}\right)+\left(\left\{\Phi_{2 N+i}\right\}-\left\{\Phi_{2 N+i-1}\right\}\right) x_{j}\right] \otimes\left[\left(\left\{\Phi_{N+i}\right\}-\left\{\Phi_{N+i-1}\right\}\right)+\left(\left\{\Phi_{2 N+i}\right\}-\left\{\Phi_{2 N+i-1}\right\}\right) x_{j}\right] \\
& \otimes\left[\left(\left\{\Phi_{N+i}\right\}-\left\{\Phi_{N+i-1}\right\}\right)+\left(\left\{\Phi_{2 N+i}\right\}-\left\{\Phi_{2 N+i-1}\right\}\right) x_{j}\right] x_{j} \\
& {[\alpha]=\left[\begin{array}{ccccc}
\alpha_{1} & -\alpha_{2} & 0 & \ldots & 0 \\
0 & \alpha_{2} & -\alpha_{3} & \ldots & 0 \\
& & \ldots & & \\
0 & 0 & 0 & \ldots & \alpha_{N}
\end{array}\right], \quad[\beta]=\left[\begin{array}{ccccc}
\beta_{1} & -\beta_{2} & 0 & \ldots & 0 \\
0 & \beta_{2} & -\beta_{3} & \ldots & 0 \\
& & \ldots & & \\
0 & 0 & 0 & \ldots & \beta_{N}
\end{array}\right]}
\end{aligned}
$$

$\left\{\Phi_{i}\right\}: i$ th row of the normalized modal matrix $[\Phi]$.

Equation (10) can be rewritten in the modal space of the underlying linear system: if the cubic coefficients of the inter-story stiffness below the $i$ th floor are constant for all the frames parallel to $x$ and $y$ axes $\left(\alpha_{i, l}=\alpha_{i}\right.$ and $\left.\beta_{i, j}=\beta_{i}\right)$, then the motion of the structure can be expressed as follows:

$$
\begin{aligned}
{[I]\{\ddot{p}\}+[2 \zeta \omega]\{\dot{p}\}+\left[\omega^{2}\right]\{p\}+[R](\{p\}} & \otimes\{p\} \otimes\{p\})= \\
-[\Gamma]\left\{\ddot{q}_{s}\right\} &
\end{aligned}
$$

$\{p\}$ : vector of modal coordinates;

$[I]$ : identity matrix;

$[2 \zeta \omega]$ : modal damping matrix (diagonal);

$\left[\omega^{2}\right]$ : modal frequencies matrix;

$[\Gamma]$ : matrix of modal participation factors;

$[R]$ : matrix of non-linearity (non-diagonal). where:

See equation (12) above.
In the presence of mild non-linearity, the change to modal space (Eq. (11)) implies two major advantages:

- the non-linear identification procedure can take advantage of previous linear identification sessions;

- the size of the problem can be significantly reduced by considering only the relevant vibration modes.

If the motion of the structure is governed by the $n_{\text {red }}$ modes, Equation (11) reduces to:

$$
\begin{array}{r}
{[I]\{\ddot{p}\}_{\text {red }}+[2 \zeta \omega]\{\dot{p}\}_{\text {red }}+\left[\omega^{2}\right]\{p\}_{\text {red }}+[R]\left(\{p\}_{\text {red }}\right.} \\
\left.\otimes\{p\}_{\text {red }} \otimes\{p\}_{\text {red }}\right)=-[\Gamma]_{\text {red }}\left\{\ddot{q}_{\mathrm{s}}\right\}
\end{array}
$$

where $[R]_{\text {red }}$ can be deduced from Equation (12) by replacing $\left\{\Phi_{i}\right\}$ with the vector $\left\{\Phi_{i}\right\}_{\text {red }}$, which contains only the relevant $n_{\text {red }}$ elements of the $i$ th row of $[\Phi]$. 

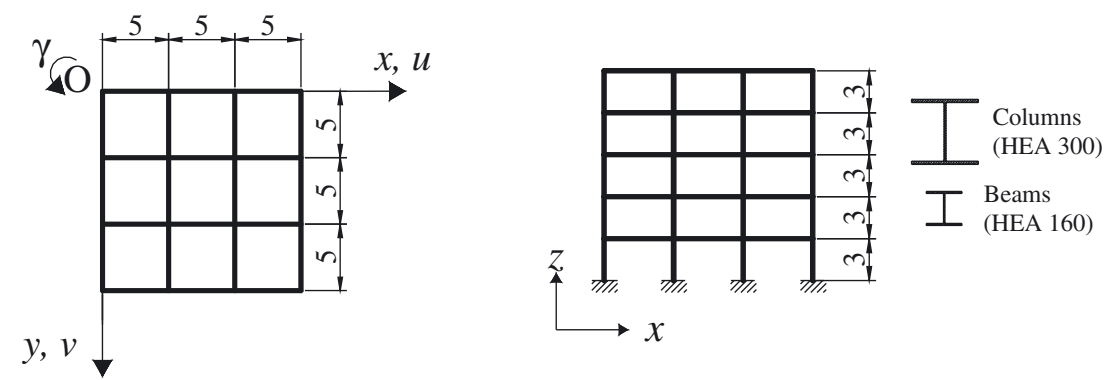

Fig. 2. Case study: steel spatial frame with eccentric masses.

Equation (13) can then be written in the ALEs form:

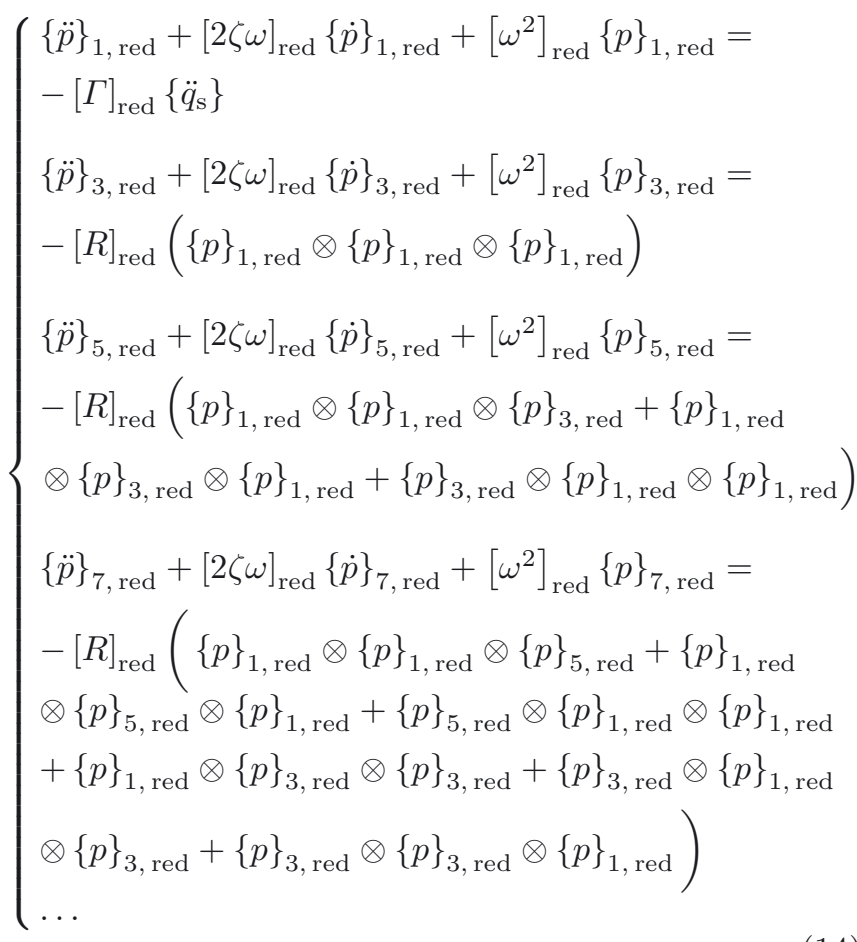

Parametric solutions referred to Equation (14), after being transformed according to Equation (4), will constitute the basis for the instantaneous parameter optimisation (Eq. (6)).

\subsection{Case study: instantaneous identification of a steel multy-story building under earthquake loading}

As a numerical application of the non-linear identification procedure, the instantaneous estimators of the dynamic properties have been determined from the seismic response of a 5-story building with eccentric masses (Fig. 2, Tab. 1). The excitation supplied to the model consisted of the E-W and N-S components ( $x$ and $y$ axes respectively) of the San Fernando earthquake (1971) as measured at the base of the Alhambra building [23] (Fig. 3). The seismic motion is supposed to produce a cubic non-linear behaviour only at the first story level, hence matrices $[\alpha]$ and $[\beta]$ assume the following expres-
Table 1. Mass eccentricities of the 5-story building.

\begin{tabular}{ccccc}
\hline & \multicolumn{2}{c}{$\begin{array}{c}\text { Position of the } \\
\text { center of mass }\end{array}$} & \multicolumn{2}{c}{$\begin{array}{c}\text { Eccentricity (\% of the } \\
\text { floor dimension) }\end{array}$} \\
\hline Story & $x(\mathrm{~m})$ & $y(\mathrm{~m})$ & $e_{x}(\%)$ & $e_{y}(\%)$ \\
1 & 7.24 & 8.5 & -1.73 & 6.67 \\
2 & 6.5 & 8.5 & -6.67 & 6.67 \\
3 & 7.58 & 7.47 & 0.53 & -0.20 \\
4 & 7.67 & 7.77 & 1.13 & 1.80 \\
5 & 6.81 & 7.65 & -4.60 & 1.00 \\
\hline
\end{tabular}

Table 2. First modal frequencies and damping ratios and nonlinear stiffnesses of the 5 -story building.

\begin{tabular}{ccc}
\hline Mode & $f(\mathrm{~Hz})$ & $\zeta(\%)$ \\
\hline $1\left(1^{\circ}\right.$ flexural $\left.x\right)$ & 0.44 & 3 \\
$2\left(1^{\circ}\right.$ flexural $\left.y\right)$ & 0.47 & 2 \\
$3\left(1^{\circ}\right.$ torsional $)$ & 0.60 & 1.5 \\
$4\left(2^{\circ}\right.$ flexural $\left.x\right)$ & 1.66 & 3.5 \\
$5\left(2^{\circ}\right.$ flexural $\left.y\right)$ & 1.85 & 4.5 \\
$6\left(2^{\circ}\right.$ torsional $)$ & 2.30 & 6 \\
$7\left(3^{\circ}\right.$ flexural $\left.x\right)$ & 3.61 & 2 \\
$8\left(3^{\circ}\right.$ flexural $\left.y\right)$ & 4.16 & 5 \\
$9\left(3^{\circ}\right.$ torsional $)$ & 5.27 & 2 \\
\hline$k_{3 x}\left(\mathrm{~N} \cdot \mathrm{m}^{-3}\right)$ & $-4 \times 10^{9}$ \\
$k_{3 y}\left(\mathrm{~N} \cdot \mathrm{m}^{-3}\right)$ & \multicolumn{2}{c}{$-1 \times 10^{10}$} \\
\hline
\end{tabular}

sion:

$$
[\alpha]=\left[\begin{array}{ccccc}
k_{3 x} & 0 & 0 & 0 & 0 \\
0 & 0 & 0 & 0 & 0 \\
0 & 0 & 0 & 0 & 0 \\
0 & 0 & 0 & 0 & 0 \\
0 & 0 & 0 & 0 & 0
\end{array}\right], \quad[\beta]=\left[\begin{array}{ccccc}
k_{3 y} & 0 & 0 & 0 & 0 \\
0 & 0 & 0 & 0 & 0 \\
0 & 0 & 0 & 0 & 0 \\
0 & 0 & 0 & 0 & 0 \\
0 & 0 & 0 & 0 & 0
\end{array}\right]
$$

where $k_{3 x}$ and $k_{3 y}$ are the cubic stiffness coefficients corresponding to the first interstory drift, respectively for $x$ and $y$ directions.

Table 2 reports the values of the principal modal frequencies and damping ratios of the building, as well as non-linear stiffness coefficients, in the assumption of cubic non-linearity (see Sect. 3); Figure 4 shows the displacement at the first story level and the corresponding non-linear force-displacement laws, respectively. From these graphs, it is evident that a non-linear response is 

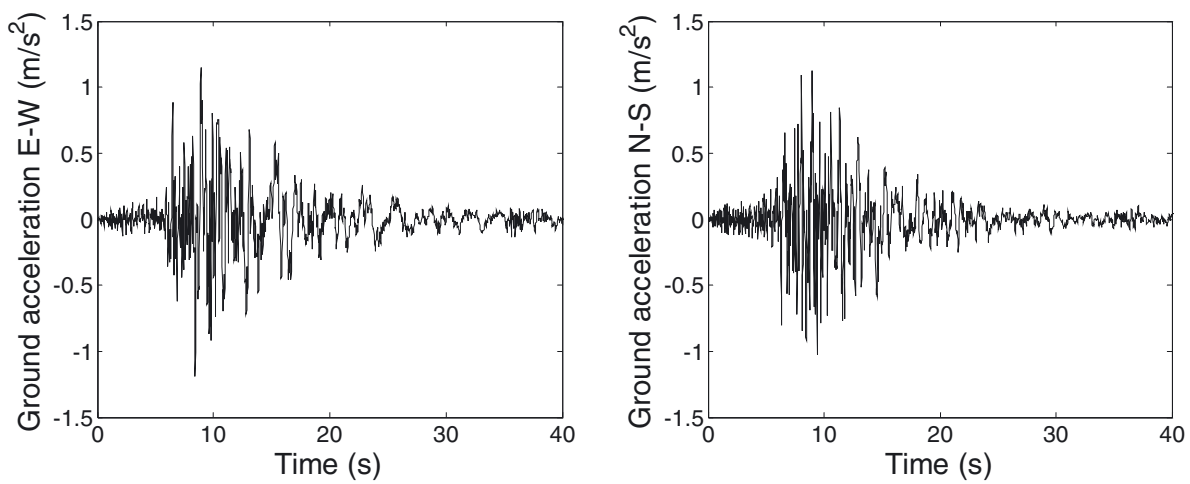

Fig. 3. Ground acceleration (San Fernando earthquake, 1971, E-W and N-S components).
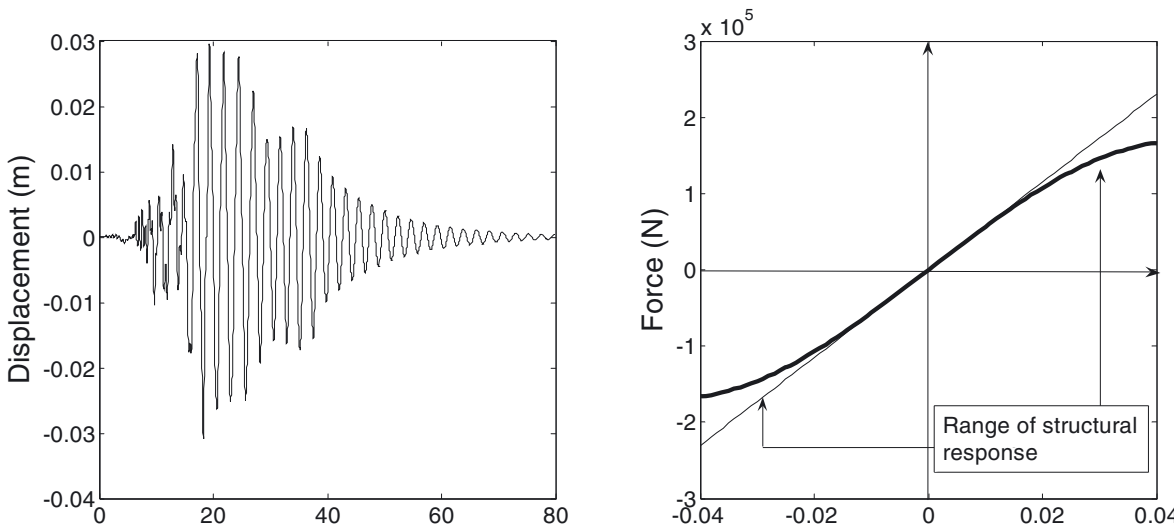

$\mathrm{x}$-axis
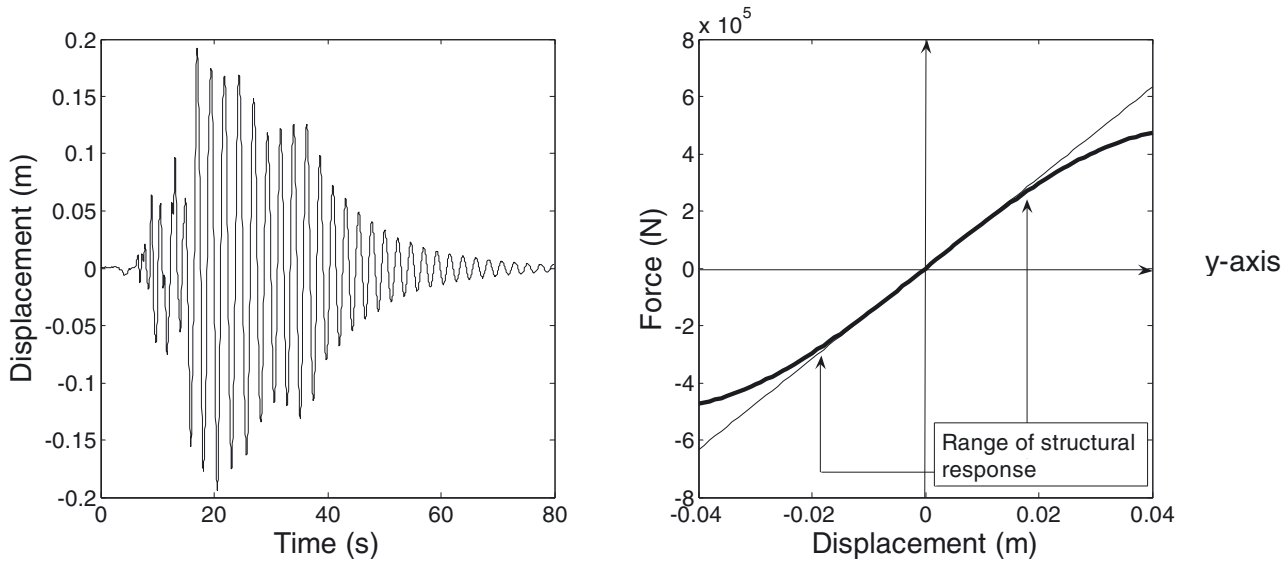

Fig. 4. Displacement at the 1st story level and force-displacement law.

induced only in the $x$ direction, while parallel to $y$, the structure exhibits a substantially linear behaviour.

It was assumed that the vibration of the system had been measured at 3rd, 4th and 5th floor level in the $x$ direction, and that the first three linear modes of the structure had been determined through a preliminary identification stage, performed on the first part of the signals. Moreover, it was assumed that an estimate of the matrix of modal participation factor was available from a numerical model of the building.

In order to investigate the influence of the accuracy of the data contained in the modal participation matrix, three simulations were performed, by introducing errors of different magnitudes (from $5 \%$ to $30 \%$ of the exact value) into coefficients $\Gamma_{i x}$ and $\Gamma_{i y}(i=1,2,3$, see Eq. (14) $)$ and adding to the output a steady Gaussian noise (RMS level of noise equal to $5 \%$ the RMS value of the measured signals).

In the assumption that the response is governed by the three lowest modes $p_{1}, p_{2}$ and $p_{3}$ (first bending modes in $x$ and $y$ direction and first torsion mode), the displacements of the first story can be expressed as follows:

$$
\left\{\begin{array}{l}
u_{1} \cong \Phi_{1,1} p_{1}+\Phi_{1,2} p_{2}+\Phi_{1,3} p_{3} \\
v_{1} \cong \Phi_{N+1,1} p_{1}+\Phi_{N+1,2} p_{2}+\Phi_{N+1,3} p_{3} \\
\gamma_{1} \cong \Phi_{2 N+1,1} p_{1}+\Phi_{2 N+1,2} p_{2}+\Phi_{2 N+1,3} p_{3}
\end{array}\right.
$$




$$
\begin{gathered}
\left\{A_{1}\right\}_{\text {red }}=\sum_{l=1}^{N_{x}}\left(\left\{\Phi_{1,1} \Phi_{1,2} \Phi_{1,3}\right\}-\left\{\Phi_{2 N+1,1} \Phi_{2 N+1,2} \Phi_{2 N+1,3}\right\} y_{l}\right) \\
\otimes\left(\left\{\Phi_{1,1} \Phi_{1,2} \Phi_{1,3}\right\}-\left\{\Phi_{2 N+1,1} \Phi_{2 N+1,2} \Phi_{2 N+1,3}\right\} y_{l}\right) \\
\otimes\left(\left\{\Phi_{1,1} \Phi_{1,2} \Phi_{1,3}\right\}-\left\{\Phi_{2 N+1,1} \Phi_{2 N+1,2} \Phi_{2 N+1,3}\right\} y_{l}\right) \\
\left\{B_{1}\right\}_{\text {red }}=\sum_{j=1}^{N_{y}}\left(\left\{\Phi_{N+1,1} \Phi_{N+1,2} \Phi_{N+1,3}\right\}+\left\{\Phi_{2 N+1,1} \Phi_{2 N+1,2} \Phi_{2 N+1,3}\right\} x_{j}\right) \\
\otimes\left(\left\{\Phi_{N+1,1} \Phi_{N+1,2} \Phi_{N+1,3}\right\}+\left\{\Phi_{2 N+1,1} \Phi_{2 N+1,2} \Phi_{2 N+1,3}\right\} x_{j}\right) \\
\otimes\left(\left\{\Phi_{N+1,1} \Phi_{N+1,2} \Phi_{N+1,3}\right\}+\left\{\Phi_{2 N+1,1} \Phi_{2 N+1,2} \Phi_{2 N+1,3}\right\} x_{j}\right) \\
\left\{D_{1}\right\}_{\text {red }}=\sum_{l=1}^{N_{x}}\left(\left\{\Phi_{1,1} \Phi_{1,2} \Phi_{1,3}\right\}-\left\{\Phi_{2 N+1,1} \Phi_{2 N+1,2} \Phi_{2 N+1,3}\right\} y_{l}\right) \\
\otimes\left(\left\{\Phi_{1,1} \Phi_{1,2} \Phi_{1,3}\right\}-\left\{\Phi_{2 N+1,1} \Phi_{2 N+1,2} \Phi_{2 N+1,3}\right\} y_{l}\right) \\
\otimes\left(\left\{\Phi_{1,1} \Phi_{1,2} \Phi_{1,3}\right\}-\left\{\Phi_{2 N+1,1} \Phi_{2 N+1,2} \Phi_{2 N+1,3}\right\} y_{l}\right) y_{l} \\
\left\{E_{1}\right\}_{\text {red }}=\sum_{j=1}^{N_{y}}\left(\left\{\Phi_{N+1,1} \Phi_{N+1,2} \Phi_{N+1,3}\right\}+\left\{\Phi_{2 N+1,1} \Phi_{2 N+1,2} \Phi_{2 N+1,3}\right\} x_{j}\right) \\
\otimes\left(\left\{\Phi_{N+1,1} \Phi_{N+1,2} \Phi_{N+1,3}\right\}+\left\{\Phi_{2 N+1,1} \Phi_{2 N+1,2} \Phi_{2 N+1,3}\right\} x_{j}\right) \\
\otimes\left(\left\{\Phi_{N+1,1} \Phi_{N+1,2} \Phi_{N+1,3}\right\}+\left\{\Phi_{2 N+1,1} \Phi_{2 N+1,2} \Phi_{2 N+1,3}\right\} x_{j}\right) x_{j}
\end{gathered}
$$

The non-linear internal forces can then be written by particularizing Equation (13) for the case of non-linearity localised only at the first story:

$$
\begin{aligned}
& \left\{\begin{array}{l}
\ddot{p}_{1} \\
\ddot{p}_{2} \\
\ddot{p}_{3}
\end{array}\right\}=\left[\begin{array}{ccc}
2 \zeta_{1} \omega_{1} & 0 & 0 \\
0 & 2 \zeta_{2} \omega_{2} & 0 \\
0 & 0 & 2 \zeta_{3} \omega_{3}
\end{array}\right]\left\{\begin{array}{l}
\dot{p}_{1} \\
\dot{p}_{2} \\
\dot{p}_{3}
\end{array}\right\}+\left[\begin{array}{ccc}
\omega_{1}^{2} & 0 & 0 \\
0 & \omega_{2}^{2} & 0 \\
0 & 0 & \omega_{3}^{2}
\end{array}\right]\left\{\begin{array}{l}
p_{1} \\
p_{2} \\
p_{3}
\end{array}\right\} \\
& +\left[\begin{array}{lll}
\Phi_{1,1} & \Phi_{N+1,1} & \Phi_{2 N+1,1} \\
\Phi_{1,2} & \Phi_{N+1,2} & \Phi_{2 N+1,2} \\
\Phi_{1,3} & \Phi_{N+1,3} & \Phi_{2 N+1,3}
\end{array}\right]\left[\begin{array}{c}
k_{3 x}\left\{A_{1}\right\}_{\text {red }} \\
k_{3 y}\left\{B_{1}\right\}_{\text {red }} \\
-k_{3 x}\left\{D_{1}\right\}_{\text {red }}+k_{3 x}\left\{E_{1}\right\}_{\text {red }}
\end{array}\right] \\
& \times\left(\left\{\begin{array}{l}
p_{1} \\
p_{2} \\
p_{3}
\end{array}\right\} \otimes\left\{\begin{array}{l}
p_{1} \\
p_{2} \\
p_{3}
\end{array}\right\} \otimes\left\{\begin{array}{l}
p_{1} \\
p_{2} \\
p_{3}
\end{array}\right\}\right)=-\left[\begin{array}{l}
\Gamma_{1 x} \Gamma_{1 y} \\
\Gamma_{2 x} \Gamma_{2 y} \\
\Gamma_{3 x} \Gamma_{3 y}
\end{array}\right]\left\{\begin{array}{l}
\ddot{q}_{x} \\
\ddot{q}_{y}
\end{array}\right\}
\end{aligned}
$$

being:

$$
\text { see equation (17) above. }
$$

In this specific application, the parameters to be identified are the modal frequencies and damping ratios of the first three modes and the cubic stiffness coefficients of the inter-story drift: the vector of instantaneous estimators, $\boldsymbol{p}(t)=\left\{\zeta_{1}(t) \zeta_{2}(t) \zeta_{3}(t) \omega_{1}(t) \omega_{2}(t) \omega_{3}(t) k_{3 x}(t) k_{3 y}(t)\right\}^{\mathrm{T}}$, was determined by resorting to the first four terms of the Volterra series (Eq. (2)). Figures 5 and 6 show the instantaneous estimators in the time interval ranging from $25 \mathrm{~s}$ to $35 \mathrm{~s}$ and Table 3 summarizes the mean characteristics of the estimators in the same interval.

In all the cases considered, modal damping and frequency instantaneous estimators proved to be robust to noise and characterized by small values of the coefficient of variation. The instantaneous estimator of $k_{3 x}$ showed mean values close to the theoretical ones and coefficients of variation that depended to a significant extent on the magnitude of the introduced errors. When the errors in the modal participation factors increase, the mean value of $k_{3 x}$ may happen to approach the exact value: this can be due to errors in the inputs acting on different degrees of freedom, which are partially compensated for by a fictitious change in the absolute value of cubic stiffness. Similar effects on $k_{3 x}$ can be produced also by the influence of modes that are not captured by the model.

It was not possible to work out an estimate of $k_{3 y}$, since, under this particular seismic motion, the nonlinearity was not appreciably activated in the N-S component of the structural response (see Fig. 4).

Even in the presence of appreciable errors in the evaluation of non-linear parameters, structural modelling based on the results of the instantaneous identification procedure resulted to be considerably improved, as can be seen from Figure 7, where the acceleration of the 5th floor in the $x$ direction (error in $\Gamma_{i} \leq 15 \%$ ) is compared to the identified response and to the response of the underlying linear system (both calculated via direct numerical integration).

In conclusion, it is worthwhile noting that earthquakes cause phenomena, including hysteresis, cracking and degradation, that are far too complex to be directly captured by Volterra time-invariant models; nevertheless, a polynomial behaviour can be assumed to summarize the global dynamics of real buildings when they are subjected to moderate loads, such as an excitation supplied during modal testing or mild earthquakes. In these circumstances, even a rough estimate of the non-linear parameters may improve dramatically the prediction capabilities of the model and/or simply detect structural anomalies and changes. 

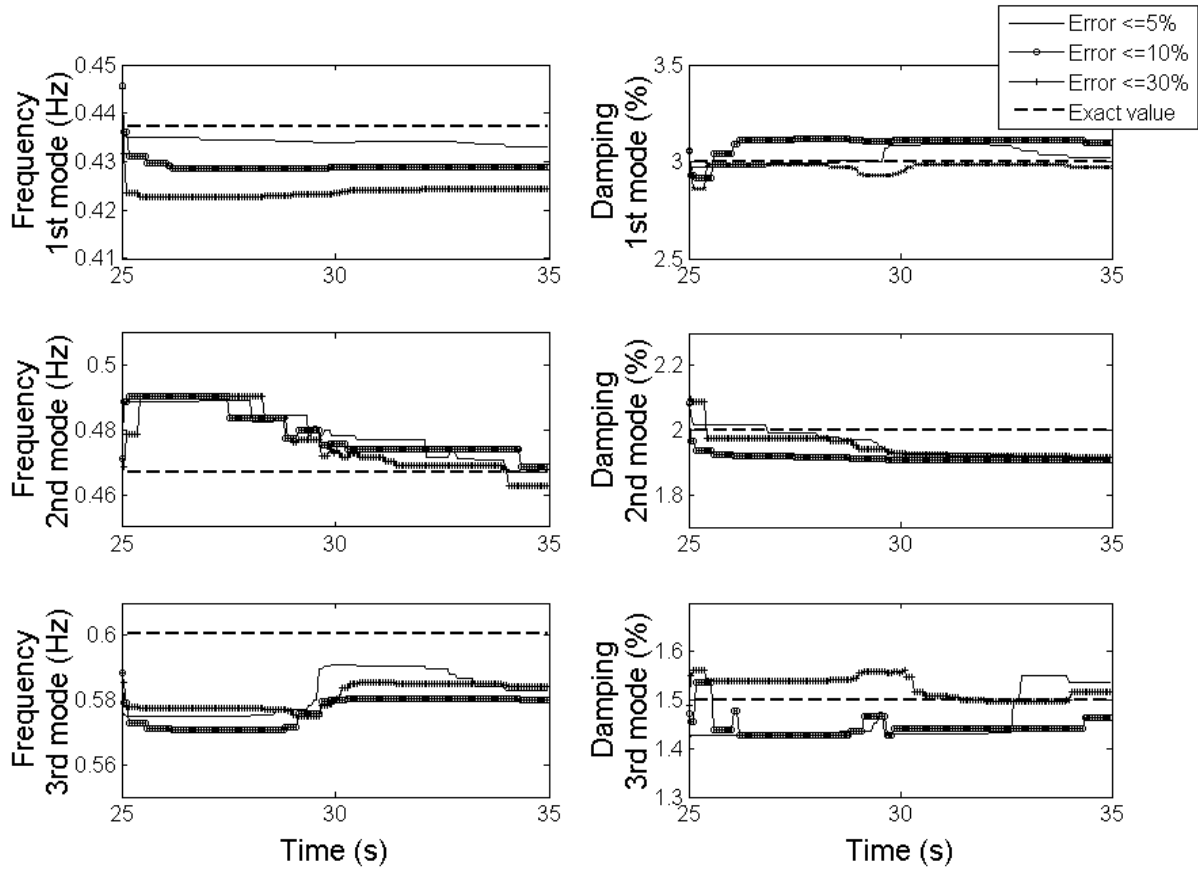

Fig. 5. Steel frame: instantaneous estimators of the modal frequencies and modal damping ratios.
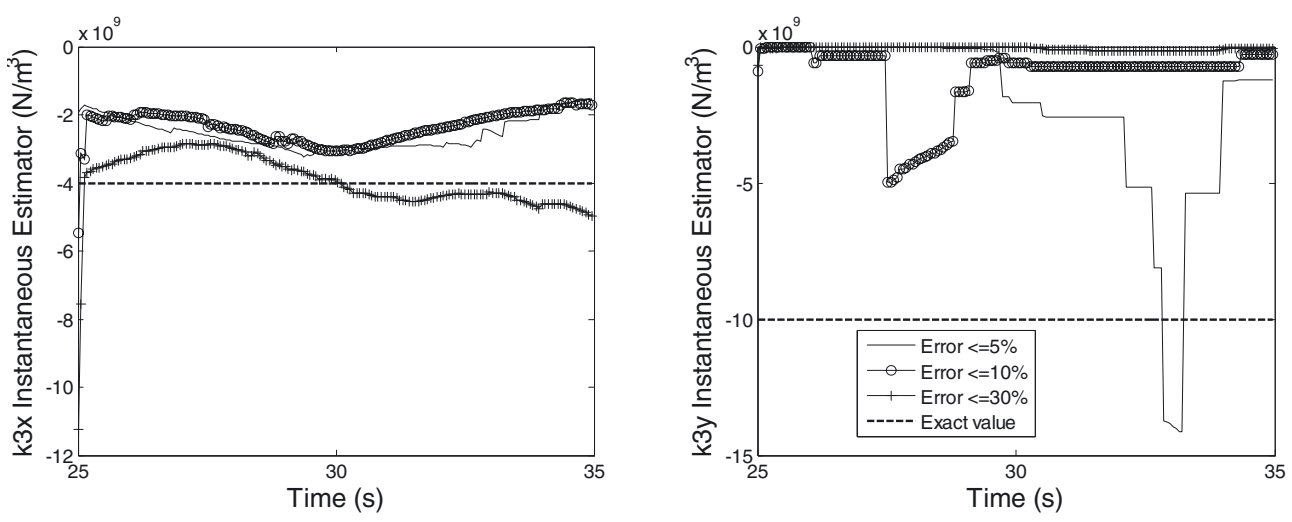

Fig. 6. Steel frame: instantaneous estimators of $k_{3 x}$ and $k_{3 y}$.

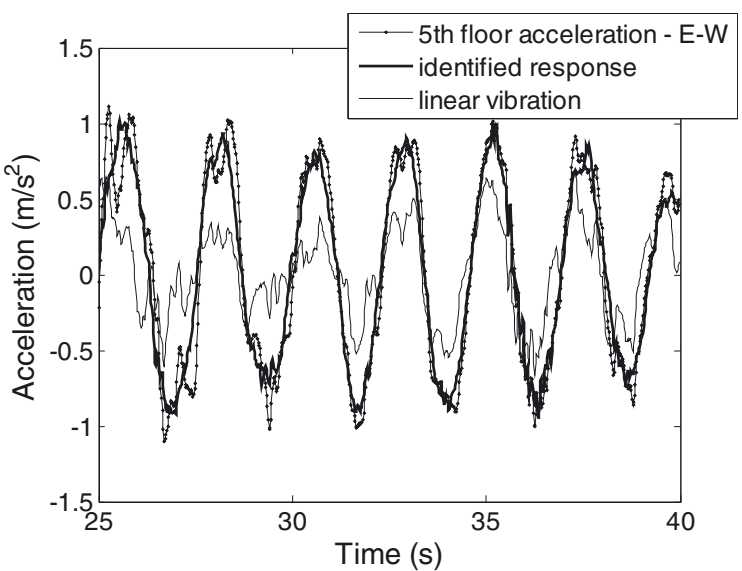

Fig. 7. Steel frame: comparison among the measured acceleration, the identified one, and the response of the underlying linear system.

\section{Conclusions}

The purpose of this paper was to propose a parametric procedure for the instantaneous identification of weakly non-linear framed structures. The identification is conducted by approximating the dynamic response through a finite Volterra series, whilst the instantaneous estimates are derived directly from a linear time-frequency representation of the signals detected. Theories and examples refer to multi-story buildings characterised by concentrated non-linearities, which were simulated by introducing polynomial stiffness laws.

The stability over time of the estimators for the parameters that define the system, such as modal frequencies, damping ratios and cubic stiffness coefficients, was analysed: the best results were obtained for the linear parameters (frequency and damping ratio), whose mean values turned out to be very close to the theoretical ones, 
Table 3. Mean characteristics of the instantaneous estimators.

\begin{tabular}{cccc}
\hline Parameter & Error in $\Gamma_{i}$ & Mean value & Coefficient of variation \\
\hline$\zeta_{1}(-)$ & $\leq 5 \%$ & 0.0303 & 0.0145 \\
& $\leq 15 \%$ & 0.0310 & 0.0187 \\
& $\leq 30 \%$ & 0.0297 & 0.0089 \\
\hline \multirow{3}{*}{$\zeta_{2}(-)$} & $\leq 5 \%$ & 0.0196 & 0.0210 \\
& $\leq 15 \%$ & 0.0191 & 0.0084 \\
& $\leq 30 \%$ & 0.0195 & 0.0195 \\
\hline & $\leq 5 \%$ & 0.0146 & 0.0323 \\
$\zeta_{3}(-)$ & $\leq 15 \%$ & 0.0144 & 0.0159 \\
& $\leq 30 \%$ & 0.0153 & 0.0144 \\
\hline & $\leq 5 \%$ & 0.4342 & 0.0014 \\
$f_{1}(\mathrm{~Hz})$ & $\leq 15 \%$ & 0.4291 & 0.0038 \\
& $\leq 30 \%$ & 0.4238 & 0.0045 \\
\hline \multirow{2}{*}{$f_{2}(\mathrm{~Hz})$} & $\leq 5 \%$ & 0.4797 & 0.0161 \\
& $\leq 15 \%$ & 0.4796 & 0.0151 \\
& $\leq 30 \%$ & 0.4769 & 0.0206 \\
\hline \multirow{2}{*}{$f_{3}(\mathrm{~Hz})$} & $\leq 5 \%$ & 0.5822 & 0.0113 \\
& $\leq 15 \%$ & 0.5765 & 0.0078 \\
& $\leq 30 \%$ & 0.5810 & 0.0068 \\
\hline \multirow{2}{*}{$k_{3 x}\left(\mathrm{~N} . \mathrm{m}^{3}\right)$} & $\leq 5 \%$ & $-2.5361 \times 10^{9}$ & 0.1831 \\
& $\leq 15 \%$ & $-2.3590 \times 10^{9}$ & 0.2058 \\
\hline \multirow{2}{*}{$k_{3 y}\left(\mathrm{~N} . \mathrm{m}^{3}\right)$} & $\leq 30 \%$ & $-3.9384 \times 10^{9}$ & 0.2304 \\
& $\leq 5 \%$ & $-2.1029 \times 10^{9}$ & .5050 \\
\hline
\end{tabular}

with very small coefficients of variation. The estimates of the non-linear parameters proved to be the most sensitive to the presence of noise and inaccuracy in the modal participation factors. This notwithstanding, the prediction capacity of the updated 3D frame model resulted to be considerably improved, as required in structural engineering applications.

It must be stressed that, in addition to computational advantages, a proper choice for the order of the Volterra series expansion can filter out measurement or integration distortions, which otherwise would be implicitly attributed to higher-order Volterra terms.

Future research will consider the extension to systems with structural degradation, as required in a vast majority of applications in the fields of structural diagnostics and earthquake engineering.

\section{References}

[1] G. Shi, S.N. Atluri, Static and dynamic analysis of space frames with non-linear flexible connections, Int. J. Num. Meth. Eng. 28 (1989) 2635-2650

[2] P.D. Moncarz, K.H. Gerstle, Steel frames with non-linear connections, J. Struct. Div. ASCE 107 (1981) 1427-1441

[3] A. Mohebkhaha, A.A. Tasnimia, H.A. Moghadamb, Nonlinear analysis of masonry-infilled steel frames with openings using discrete element method, J. Constr. Steel Res. 64 (2008) 1463-1472
[4] N.M.M. Maia, J.M.M. Silva, Theoretical and Experimental Modal Analysis, Research Study Press LTD, Taunton, England, 1997

[5] J.K. Hammond, P.R. White, The analysis of nonstationary signals using time-frequency methods, J. Sound Vib. 190 (1996) 419-447

[6] S. Mallat, G. Papanicolaou, Z. Zhang, Adaptive covariance estimation of locally stationary processes, Ann. Stat. 28 (1998) $1-47$

[7] R. Ceravolo, Time-frequency analysis, in Encyclopedia of Structural Health Monitoring, C. Boller, F.-K. Chang \& Y. Fujino (eds.), Wiley \& Sons, 2008

[8] P. Bonato, R. Ceravolo, A. De Stefano, F. Molinari, Use of cross time-frequency estimators for the structural identification in non-stationary conditions and under unknown excitation, J. Sound Vib. 237 (2000) 775-791

[9] R. Ceravolo, Use of instantaneous estimators for the evaluation of structural damping, J. Sound Vib. 274 (2004) 385-401

[10] S. Erlicher, P. Argoul, Modal identification of linear nonproportionally damped systems by wavelet transform, Mech. Sys. Sig. Proc. 21 (2007) 1386-1421

[11] W.B. Collis, P.R. White, J.K. Hammond, Higher-order spectra: the bispectrum and trispectrum, Mechanical Systems and Signal Processing 12 (1998) 375-394

[12] S.B. Kim, E.J. Powers, Estimation of Volterra kernels via higher-order statistical signal processing, Chapter 7 in Higher-Order Statistical Signal Processing B. Boashash, E.J. Powers, A. Zoubir (eds.), Longman/Wiley, Melbourne and New York, 1995 
[13] G.V. Demarie, R. Ceravolo, A. De Stefano, Instantaneous identification of polynomial non-linearity based on Volterra series representation, Key Engineering Materials 293-294 (2005) 703-710

[14] K. Worden, G. Manson, G.R. Tomlinson, A harmonic probing algorithm for the multi-input Volterra series, J. Sound Vib. 201 (1997) 67-84

[15] K. Worden, G. Manson, G.R. Tomlinson, Random vibration of a multi-degree-of-freedom non-linear system using the Volterra series, J. Sound Vib. 226 (1999) 397-405

[16] F. Thouverez, L. Jezequel, Identification of a localized non-linearity, Int. J. Non-Lin. Mech. 33 (1998) 935-945

[17] I. Tawfiq, T. Vinh, Contribution to extension of modal analysis to non-linear structure using Volterra series, Mechanical Systems and Signal Processing 17 (2003) 379407
[18] A. Chatterjee, N.S. Vyas, Non-linear parameter estimation in multi-degree-of-freedom systems using multiinput Volterra series, Mechanical Systems and Signal Processing 18 (2004) 457-489

[19] M. Schetzen, The Volterra/Wiener theories of non-linear systems, Krieger publishing company, Malabar, FL, 1980

[20] W.J. Rugh, Nonlinear system theory. The Volterra/Wiener approach, 2002 (Web version: http://www.ece.jhu.edu/〜rugh/volterra/book.pdf)

[21] J.A. Vazquez Feijoo, K. Worden, R. Stanway, Associated linear equations for Volterra operators, Mechanical Systems and Signal Processing 19 (2005) 57-69

[22] J.R. Carson, Notes on the theory of modulation, Proc. IEEE 10 (1922) 57-64

[23] W.K. Cloud, D.E. Hudson, Strong motion data from San Fernando, California, earthquake of February 9, 1971, California Division of Mines and Geology Bull. 196 (1975) 273-303 